\title{
Wearable motion sensors and digital biomarkers in stroke rehabilitation
}

\author{
Adrian Derungs, Chair of Digital Health, Friedrich-Alexander University Erlangen-Nürnberg, Erlangen, Germany, \\ adrian.derungs@fau.de \\ Corina Schuster-Amft, Research Department, Reha Rheinfelden, Rheinfelden, Switzerland, \\ Institute for Rehabilitation and Performance Technology, Bern University of Applied Sciences, Burgdorf, Switzerland, \\ Department of Sport, Exercise and Health, University of Basel, Basel, Switzerland, C.Schuster@ reha-rhf.ch \\ Oliver Amft, Chair of Digital Health, Friedrich-Alexander University Erlangen-Nürnberg, Erlangen, Germany, \\ oliver.amft@fau.de
}

\section{Introduction}

Wearable motion sensors and personalised digital biomarkers could revolutionise stroke rehabilitation. In this work, we propose three novel digital biomarkers for the longitudinal performance monitoring and movement evaluation of hemiparetic patients after stroke that could be used in free-living.

\section{Methods}

We introduce convergence points (CP) as a marker family that describe the relation of motion between body sides across time to predict a virtual recovery point using regression techniques. The regression-based $\mathrm{CP}$ estimation interprets continuously recorded IMU data, i.e. gait parameters, including stride count and stride duration, which are itself relevant for evaluating the walking abilities. The CP's, expressed in days, can help clinicians to personalise patient training, e.g. treadmill walking or balance exercises. With personalised models for estimating the physical activity (PA), we analysed differences in the affected and less-affected upper and lower body sides' movement intensity that are mapped to energy levels and expressed in metabolic equivalents. Another novel digital biomarker that can support clinicians are 3D posture cubics to quantify and visualise the functional range of motion (fROM) during therapy and free-living.

\section{Results}

In an observational clinical study, including 11 outpatients after stroke, we derived more than 620 hours of annotated movement data to investigate activities in therapy and free-living. CP estimates revealed inter-patient variability within and across gait parameters and that the patient behaviour was influenced by individual therapy schedules. The PA analysis showed differences between the affected and less-affected body sides and the upper an lower body and that patients tend to sedentary behaviour. The fROM analysis using posture cubics confirmed that the upper arm range of motion depends on the rehabilitation context, specifically, if patients perform therapy exercises or activities of daily living.

\section{Conclusion}

Our digital biomarkers, which are specifically designed for longitudinal stroke rehabilitation, hold promise for applications in free-living. 


\section{Wearable Impedance Pneumography}

Michael Klum, Chair of Electronics and Medical Signal Processing, Technische Universität Berlin, Einsteinufer 17, 10587 Berlin, Germany, michael.klum@tu-berlin.de

Mike Urban, Alexandru-Gabriel Pielmus, Reinhold Orglmeister: Chair of Electronics and Medical Signal Processing, Technische Universität Berlin, Einsteinufer 17, Berlin, info@emsp.tu-berlin.de

\section{Introduction}

Respiratory diseases are one of the leading causes of deaths worldwide. The prevalence of sleep apnea, its cardiovascular consequences, postoperative respiratory instability and severe respiratory syndromes further highlight the importance of respiratory monitoring. Typical methods, however, rely on obtrusive nasal cannulas and belts. Impedance pneumography (IP) is a promising bioimpedance application, which aims to estimate respiratory parameters from the thorax impedance. Currently, IP configurations require large inter-electrode distances, diminishing its applicability in a wearable context. We propose an IP configuration with $55 \mathrm{~mm}$ spacing using our recently presented sensor patch.

\section{Methods}

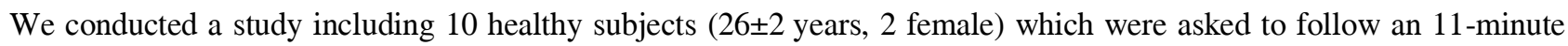
long breathing sequence in the supine, lateral and prone position. Using our multimodal, digital stethoscope patch, we recorded a $55 \mathrm{~mm}$ IP signal on the upper right thorax. Simultaneously, a $300 \mathrm{~mm}$ IP on the left half-thorax was acquired using the Osypka Medical GmbH ICON-Core ${ }^{\mathrm{TM}}$ monitor. Using the SOMNOmedics GmbH SOMNO HD ${ }^{\mathrm{TM}}$ system, a respiratory reference was acquired using a pneumotachometer and facial mask. We employed time-delay neural networks (TDNN) with two to three hidden layers, 5 to 15 neurons each and input delay vectors from 1 to 30 taps as regressors. Models were selected using the Bayesian information criterion.

\section{Results}

Using TDNNs, respiration rate (RR) errors below 1 breaths per minute, flow correlations of 0.81 and relative flow errors of $38 \%$ were achieved for the $55 \mathrm{~mm}$ IP. Transients in the respiratory signal were tracked, even though with less accuracy than in the $300 \mathrm{~mm}$ setup. We found position-dependent performances, with the prone position being the least optimal.

\section{Conclusion}

We conclude that high accuracy RR estimation is possible in a $55 \mathrm{~mm}$ IP configuration. Respiratory flow can be roughly estimated. Further research combining several biosignals for a more robust, wearable flow estimation is recommended. 


\section{Measurement of respiratory rate with inertial measurement units}

Simon Beck, Institute of Technical Medicine, Furtwangen University, Villingen-Schwenningen, Germany, simon.beck@hs-furtwangen.de

Bernhard Laufer, Sabine Krueger-Ziolek, Knut Moeller, Institute of Technical Medicine, Furtwangen University, Villingen-Schwenningen, Germany www.item.hs-furtwangen.de

\section{Introduction}

Due to demographic change, increasing air pollution and increasing incidence if allergies, more and more people are suffering from lung or other respiratory diseases which need to be monitored. New methods to speed up the process of time-consuming bodyplethysmograph tests would be very cost-saving, could increase the number of possible tests or make them even possible when considering old people, children and people with pre-existing conditions.

\section{Methods}

The relative angle between the dorsal and the ventral thorax is measuretd by using Kalman-filtered quaternions, given by low-cost inertial measurement units (IMUs). The IMU-data and a parallel reference measurement from a medical spirometer is first processed with a microcontroller, then filtered with a Butterworth-bandpass-filter $10^{\text {th }}$ order and finally the main frequency is found by using FFT with MATLAB.

Beginning with normal breath rate and then increasing it in two steps for 30 seconds each, 5 participants followed a 3 step breathing maneuver. Focus was on breast breathing and the measurement was done in a sitting position.

\section{Results}

The butterworth-filtering could filter out most of the noise and disturbances due to motion artefacts.

The Method is able to measure the respiratory rate $<30$ breaths per minute with a deviation of less than $0.2 /$ min. Above that mark, a deviation with less than 0.8 breaths per minute sill allows accurate measurement.

With a frequency rage from 6-90 breaths per minute the IMU-belt could be used even in extreme sports, where very high respiratory rates occur.

\section{Conclusion}

The tested IMU-based measurement of the respiratory rate is possible with low-cost inertial measurement units. Furthermore are the results very precise and, combined with an ECG, could provide a system for surveillance of the main vital parameters. A further goal is to implement a method to evaluate the breathing volume while breathing. 


\section{An alternative way to measure total lung capacity: a pilot study}

Bernhard Laufer, Institute of Technical Medicine (ITeM), Furtwangen University, Villingen-Schwenningen, Germany, b.laufer@hs-furtwangen.de Paul D. Docherty, University of Canterbury, Christchurch, New Zealand

Sabine Krueger-Ziolek, Institute of Technical Medicine (ITeM), Furtwangen University, Villingen-Schwenningen, Germany

Fabian Hoeflinger, University of Freiburg, Freiburg, Germany

Leonhard Reindl, University of Freiburg, Freiburg, Germany

Knut Moeller, Institute of Technical Medicine (ITeM), Furtwangen University, Villingen-Schwenningen, Germany

\section{Introduction}

Respiratory parameters are important for analysis and treatment of critically ill patients, which has led to the development of various measurement systems, such as spirometry, optoelectronic plethysmography OEP or body plethysmography. But, if the total lung capacity TLC is of interest, spirometry and OEP fails. In this study, a new method to measure the total lung capacity is introduced.

\section{Methods}

The introduced TLC-device based on Boyle Mariotte law, which states that the product of pressure and volume is constant. The device is a small tube with an inspiration valve at the end and an integrated digital pressure sensor. To measure volume changes of the upper body, an OEP system was used. As reference system for TLC determination a body plethysmograph was utilized.

This feasibility study was undertaken with 3 lung healthy subjects. After the reference measurement of the TLC (body plethysmograph), the subject breathed normally for about 30 seconds. Afterwards, at start of inhalation the subject used the TLC-device to inhale maximally through the inspiration valve and hold the air for more than 2 seconds. The subsequent exhalation was blocked by the inspiration valve, while mouth pressure and volume change of the upper body was measured.

\section{Results and}

This pilot study showed that the TLCs obtained by the introduced device differs less than 0.4L from the total lung capacity of the body pletysmograph and that the measurement setup (OEP + TLC device) is able to measure TLC values.

\section{Conclusion}

This study showed that the augmented OEP approach can be used to obtain the total lung capacity. Further developments using a smart shirt to determine volume changes combined with the introduced TLC-device have the potential to be a non invasive, cost effective and wearable alternative for TLC determination. 


\title{
Patient Benefactor Linker
}

Hira Zahid

Ziauddin University Faculty of Engineering Science and Technology, Karachi, PAKISTAN

Afshan Saad

PAF- Karachi Institute of Economics And Technology, Karachi, PAKISTAN

Marissa Jerome

Ziauddin University Faculty of Engineering Science and Technology, Karachi, PAKISTAN

Rida Batool

Ziauddin University Faculty of Engineering Science and Technology, Karachi, PAKISTAN

Anum Muneer

Ziauddin University Faculty of Engineering Science and Technology, Karachi, PAKISTAN

Sidra Abid Syed

Ziauddin University Faculty of Engineering Science and Technology, Karachi, PAKISTAN

\begin{abstract}
This paper discusses a new evolution in the healthcare sector through a device by investigating the principle application of Artificial Neural Networks(ANN) for the selection of an optimal benefactor-donor match in organ transplantation. The device aim's to correlate $\mathrm{ABO}$ blood type, age and bone density of healthy subjects. Methods: Firstly, the linker phase integrates a light intensity(lux) meter and an RGB Color Sensor module to perform an experimental observation of agglutination of RBC's which is measured through a halogen illumination source that measures the light intensity which is displayed on a screen through the microprocessor interface. Secondly, we aim to study the possibility of quantifying calcium by using near-infrared spectroscopy to estimate bone density which involves the use of an emitting source and a photodiode as a detector/receiver. At last, the device involves designing an Artificial Neural Network (ANN) model using the MATLAB software's Neural Network Toolbox to find the optimal network architecture suitable for the analysis. This architecture is achieved by simulating different Artificial Neural Network (ANN) configurations. A database was created through an intensive survey of benefactor profiles. Conclusion: The results generated by ANN are promising for identifying optimal benefactor and patient matches. Significance: This approach has potential benefits as an increase in the number of input and parameters will provide better matches and risk associated with human error are reduced. The network can further be modeled to predict survival rates.
\end{abstract}

